\title{
Optical properties and transformation mechanism of oxygen centres and their aggregates in $\mathrm{CaF}_{2}$ crystals
}

\author{
A.S. Mysovsky ${ }^{*},{ }^{1}$, E.A. Radzhabov ${ }^{1}$, M. Reichling ${ }^{2}$, A.L. Shluger ${ }^{3}$, and P.V. Sushko ${ }^{3}$ \\ ${ }^{1}$ Vinogradov Institute of Geochemistry, 1a Favorsky St., Irkutsk 664033, Russia \\ ${ }^{2}$ Department Chemie, Universität München, Butenandtstrasse 5-13, 81377 München, Germany \\ ${ }^{3}$ Department of Physics \& Astronomy, University College London, Gower St., London WC1E 6BT, UK
}

Received 11 July 2004, revised 29 July 2004, accepted 30 July 2004

Published online 20 January 2005

PACS 61.72.Bb, 61.72.Cc, 61.72.Ji, 66.30.Jt, 71.15.Mb

Oxygen-vacancy dipoles and dimers in $\mathrm{CaF}_{2}$ crystals have been studied ab initio at DFT level and with the shell model using pair potentials. The calculated dipole reorientation barrier is $0.64 \mathrm{eV}$ and the activation energy for diffusion of the dipoles is $1.61 \mathrm{eV}$. Optical absorption of $\mathrm{O}^{2-}-\mathrm{V}_{\mathrm{A}}$ dipole have been calculated with TD DFT and identified with experimental absorption bands, which appeared to have complex structure. The photodissociation mechanism of the dipole is discussed. Several configurations of the dimer $\left(\mathrm{O}^{2-}-\mathrm{V}_{\mathrm{A}}\right)_{2}$ were calculated. The association energy for the most favourable one is $0.48 \mathrm{eV}$.

(C) 2005 WILEY-VCH Verlag GmbH \& Co. KGaA, Weinheim

1 Introduction Single crystals of $\mathrm{CaF}_{2}$ and defects in these crystals have been a topic of research for nearly 50 years. Certain aspects of this research recently gained tremendous interest in the context of the use of $\mathrm{CaF}_{2}$ as an optical material for the deep ultraviolet (DUV) and vacuum ultraviolet (VUV) spectral regions. One of the big unsolved problems in this context is oxygen contamination in the bulk and at the surface of $\mathrm{CaF}_{2}$ crystals. Oxygen is readily incorporated into the bulk during crystal growth and it is very difficult to avoid this contamination or to remove contaminants after growth. Oxygen also penetrates into the crystal upon exposure of its surface to ambient air. Extensive work on oxygen doped $\mathrm{CaF}_{2}$ has been performed to clarify the structure and diffusion of single bulk oxygen defects and their clustering into larger complexes.

In present paper we intend, firstly, to clarify the nature of optical absorption and photodissociation mechanism of oxygen-vacancy dipoles and, secondly, to study the aggregation of the dipoles.

2 Calculation details We use a hybrid ab initio - classical method, which allows to combine an accurate quantum mechanical calculation of a small cluster (QM cluster) with a cruder shell model representation of the rest of crystal. To achieve that, the system is divided into several regions:

1) QM cluster, electronic structure of which is being calculated ab initio (with B3LYP functional).

2) Classical region (usually about several hundreds of atoms), where the atoms are represented by the shell model and interact with each other and with QM atoms via pair potentials. The atomic coordinates of both QM and classical atoms can be optimised with respect to minimization of total energy of the system (geometry optimisation).

3) Region of fixed atoms surrounding classical region (up to several thousands of them). They are represented as point charges to provide the correct Madelung field inside the QM+classical region. 
The described ideology was implemented in the GUESS computer code and tested for several systems [5-7]. Apart from ab initio results reported in this work a number of calculations were performed classically with pair potentials.

3 Oxygen-vacancy dipole Two possible configurations of $\mathrm{O}^{2-}-\mathrm{V}_{\mathrm{A}}$ dipole $-<100>$ and $<110>$ configurations - have been calculated.. They are shown in Fig. 1 together with a picture of lattice distortion. The $\langle 100\rangle$ configuration is energetically the favourable one, while the $<110\rangle$ configuration has the total energy higher by

$$
\mathrm{E}\left(\mathrm{O}^{2-}-\mathrm{V}_{\mathrm{A}}\right)_{<110>}=0.45 \mathrm{eV} \text {. }
$$

The energy barrier for transformation of the $<100\rangle$ configuration into $<110\rangle$ is $0.64 \mathrm{eV}$ and the barrier for the reverse transformation is $0.15 \mathrm{eV}$. The value of $0.64 \mathrm{eV}$ turns out to be also the activation energy of $\mathrm{O}^{2-}-\mathrm{V}_{\mathrm{A}}$ dipole reorientation, because the reorientation should follow a $\langle 100>\rightarrow<110>\rightarrow<100>$ path. One could have imagined a direct transition between two differently oriented $\langle 100\rangle$ configurations, but that would mean a vacancy jump along face diagonal, i.e. in the $<110>$ direction. Such a process has a very high energy barrier - our value obtained from a classical pair potentials calculation is $2.06 \mathrm{eV}$. Therefore the only possible way of reorientation is a two-stage process. According to the conclusion of Jacobs and Ong [8], who studied thermostimulated depolarisation (TSD) in $\mathrm{CaF}_{2}: \mathrm{O}^{2-}$, the barrier for oxygen-vacancy dipole reorientation is $0.47 \mathrm{eV}$. However, this value was obtained from analysis of the TSD peak shape with the assumption that it is a simple dipole reorientation peak. We have seen this is not the case because the reorientation has two stages. In a corrected model of two-stage process the value of activation energy obtained from the same experimental data may be slightly different. It seems reasonable it should be larger than the barrier for a free anion vacancy jump.

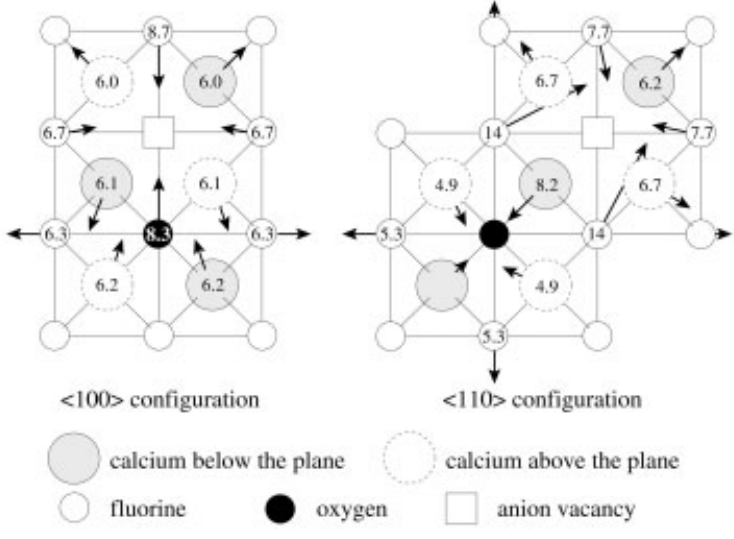

Fig. $1 \mathrm{O}^{2-}-\mathrm{V}_{\mathrm{A}}$ dipole in $<100>$ and $<110>$ configurations. The direction of displacements is shown with arrows, the values are written in circles in \% of fluorine-fluorine distance $a=2.76 \AA$.

Diffusion of the dipoles should include two types of movement: reorientation of the vacancy discussed above and an oxygen jump into the vacant site. The second type of movement has a higher energy barrier and thus determines the activation energy of $\mathrm{O}^{2-}-\mathrm{V}_{\mathrm{A}}$ diffusion. The calculated barrier is:

$$
\mathrm{E}_{\mathrm{D}}\left(\mathrm{O}^{2-}-\mathrm{V}_{\mathrm{A}}\right)=1.61 \mathrm{eV} \text {. }
$$

Optical absorption of the $\mathrm{O}^{2-}-\mathrm{V}_{\mathrm{A}}$-centre was calculated using time-dependent DFT (TD DFT). The three highest occupied molecular orbitals in the one-electron spectrum are essentially $2 p$-orbitals of the oxygen ion. One of them is $\sigma$-like (that directed along the dipole axis) and will be denoted as $\sigma\left(\mathrm{O}^{2-}\right)$, the other two are $\pi$-like and we denote them as $\pi_{1}\left(\mathrm{O}^{2-}\right)$ and $\pi_{2}\left(\mathrm{O}^{2-}\right)$. The states $\pi_{1}$ and $\pi_{2}$ are not degenerate. The lowest virtual orbital is the $1 s$-orbital of vacancy (denoted as $\sigma_{1}\left(\mathrm{~V}_{\mathrm{A}}\right)$ ). The next four unoccupied states $\left(\sigma_{2,3}\left(\mathrm{~V}_{\mathrm{A}}\right)\right.$ and $\left.\pi_{1,2}\left(\mathrm{~V}_{\mathrm{A}}\right)\right)$ are formed by a linear combination of the $2 s$ - and $2 p$-states of the vacancy. Finally, $\sigma_{4}\left(\mathrm{~V}_{\mathrm{A}}\right)$ is formed by contributions of the $3 s$ - and $3 d$ vacancy levels. 
All calculated optical transitions with oscillator strength $>0.005$ are listed in Table 1 . The transitions are clearly split into three groups. The first one contains $5.22,5.38$ and $5.61 \mathrm{eV}$ transitions from $2 p$ orbitals of oxygen to $\sigma_{1}\left(\mathrm{~V}_{\mathrm{A}}\right)$ (or ground state of vacancy). These energies are in reasonable agreement with the experimental $6.7 \mathrm{eV}$ absorption band of $\mathrm{CaF}_{2}: \mathrm{O}^{2-}[1,2]$. Although the separation between 1st and 3rd transitions is about $0.4 \mathrm{eV}$, we suggest all three of them contribute to the same band. The results of polarisation of luminescence measurements [10] also show the $6.7 \mathrm{eV}$ absorption band is complex and consists of at least two elementary peaks with separation of $0.48 \mathrm{eV}$.

Transitions $7.10-7.46 \mathrm{eV}$ from $2 p$-states of oxygen to $2 s$-, $2 p$-states of the vacancy form a second group, which can be identified with the second experimental absorption band of $\mathrm{CaF}_{2}: \mathrm{O}^{2-}$ at $8.4 \mathrm{eV}[1,2]$. The total oscillator strength of the second group is comparable with that of the first group. Finally, the 8.01, $8.17 \mathrm{eV}$ transitions $2 \mathrm{p}\left(\mathrm{O}^{2-}\right) \rightarrow 3 \mathrm{~s}, 3 \mathrm{~d}\left(\mathrm{~V}_{\mathrm{A}}\right)$ contribute to the band identified with the $9.2 \mathrm{eV}$ experimental band [2].

Table 1 Optical absorption of oxygen-vacancy dipole.

\begin{tabular}{llllll}
\hline \multicolumn{3}{c}{ configuration $<100>$} & \multicolumn{3}{c}{ configuration $<110>$} \\
transition & $\mathbf{E}, \mathbf{e V}$ & $\mathbf{f}_{\text {ose }}$ & transition & $\mathbf{E}, \mathbf{e V}$ & $\mathbf{f}_{\text {osc }}$ \\
\hline$\pi_{1}(\mathrm{O}) \rightarrow \sigma_{1}\left(\mathrm{~V}_{\mathrm{A}}\right)$ & 5.22 & 0.022 & $\sigma(\mathrm{O}) \rightarrow \sigma_{1}\left(\mathrm{~V}_{\mathrm{A}}\right)$ & 5.36 & 0.031 \\
$\pi_{2}(\mathrm{O}) \rightarrow \sigma_{1}\left(\mathrm{~V}_{\mathrm{A}}\right)$ & 5.38 & 0.010 & $\pi_{1}(\mathrm{O}) \rightarrow \sigma_{1}\left(\mathrm{~V}_{\mathrm{A}}\right)$ & 5.40 & 0.012 \\
$\sigma(\mathrm{O}) \rightarrow \sigma_{1}\left(\mathrm{~V}_{\mathrm{A}}\right)$ & 5.61 & 0.122 & $\pi_{2}(\mathrm{O}) \rightarrow \sigma_{1}\left(\mathrm{~V}_{\mathrm{A}}\right)$ & 5.45 & 0.008 \\
& & & & & \\
$\pi_{1}(\mathrm{O}) \rightarrow \sigma_{2}\left(\mathrm{~V}_{\mathrm{A}}\right)$ & 7.10 & 0.045 & $\sigma(\mathrm{O}) \rightarrow \sigma_{2}\left(\mathrm{~V}_{\mathrm{A}}\right)$ & 6.70 & 0.034 \\
$\pi_{2}(\mathrm{O}) \rightarrow \sigma_{2}\left(\mathrm{~V}_{\mathrm{A}}\right)$ & 7.25 & 0.038 & $\pi_{1}(\mathrm{O}) \rightarrow \sigma_{2}\left(\mathrm{~V}_{\mathrm{A}}\right)$ & 6.77 & 0.075 \\
$\sigma(\mathrm{O}) \rightarrow \sigma_{2}\left(\mathrm{~V}_{\mathrm{A}}\right)$ & 7.26 & 0.031 & $\pi_{2}(\mathrm{O}) \rightarrow \sigma_{2}\left(\mathrm{~V}_{\mathrm{A}}\right)$ & 6.80 & 0.036 \\
$\sigma(\mathrm{O}) \rightarrow \pi_{1}\left(\mathrm{~V}_{\mathrm{A}}\right)$ & 7.46 & 0.012 & $\sigma(\mathrm{O}) \rightarrow \sigma_{3}\left(\mathrm{~V}_{\mathrm{A}}\right)$ & 7.47 & 0.008 \\
& & & & & \\
$\pi_{1}(\mathrm{O}) \rightarrow \sigma_{4}\left(\mathrm{~V}_{\mathrm{A}}\right)$ & 8.02 & 0.017 & & & \\
$\sigma(\mathrm{O}) \rightarrow \sigma_{4}\left(\mathrm{~V}_{\mathrm{A}}\right)$ & 8.17 & 0.026 & & & \\
\hline
\end{tabular}

Photodissociation of the dipole occurs during irradiation into the 2nd and 3rd absorption bands (8.4 and $9.2 \mathrm{eV}$ ). It was suggested [3] that the dipole decays into an $\mathrm{O}^{-}$ion and an F-centre:

$$
\mathrm{O}^{2-}-\mathrm{V}_{\mathrm{A}} \rightarrow \mathrm{O}^{-}+\mathrm{F} \text {. }
$$

It should be noted, however, that the diffusion barrier for F-centres is $1.69 \mathrm{eV}$ according to [4]. This means that at a temperature of $200 \mathrm{~K} \mathrm{~F}$-centres cannot be mobile and, consequently, the above reaction of $\mathrm{O}^{2-}-\mathrm{V}_{\mathrm{A}}$ dipole dissociation is impossible at such temperatures. The photodissociation mechanism should be more complex and include the following steps (Fig. 2): 1) excitation in the 2nd or 3rd band leads to charge transfer $\mathrm{O}^{2-} \rightarrow \mathrm{V}_{\mathrm{A}}$ and an electron moves into $2 s$ - or $2 p$-state of the vacancy, i.e. $\mathrm{O}^{2-}-\mathrm{V}_{\mathrm{A}}$ dipole becomes $\mathrm{O}^{-}$-F-centre, where the F-centre is in its excited state; 2 ) since the excited states of the Fcentre are situated close to the bottom of conduction band (CB), the electron can be thermoionised at temperatures above $200 \mathrm{~K} ; 3$ ) after that the defect remaining is $\mathrm{O}^{-}-\mathrm{V}_{\mathrm{A}}$. The $\mathrm{O}^{-}$ion is not charged with respect to crystal lattice, and there is no more coulomb attraction between oxygen and vacancy (as it was for the $\mathrm{O}^{2-}-\mathrm{V}_{\mathrm{A}}$ dipole), thus the vacancy can move away from $\mathrm{O}^{-}$and become free.

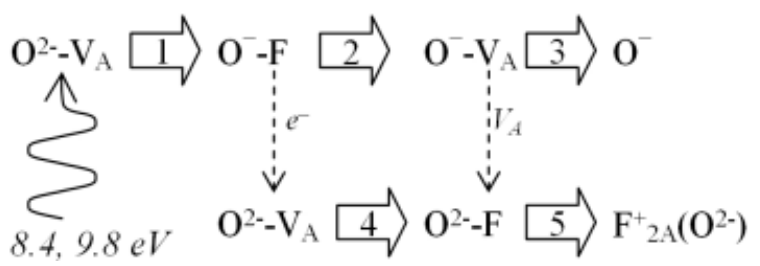

Fig. 2 Schematic view of $\mathrm{O}^{2-}-\mathrm{V}_{\mathrm{A}}$ dipole photodissociation process 
Concerning the electron which was driven into $\mathrm{CB}$ at step 2, it is then trapped on some defect, possibly another $\mathrm{O}^{2-}-\mathrm{V}_{\mathrm{A}}$ dipole. In that case the following transformations will take place: 4) another $\mathrm{O}^{2-}-\mathrm{V}_{\mathrm{A}}$ dipole traps the electron and becomes an $\mathrm{F}_{\mathrm{A}}\left(\mathrm{O}^{2-}\right)$-centre; 5) this centre can trap one of the free vacancies which are created in photodissociation (at steps 1-3); then it turns into an $\mathrm{F}_{2 \mathrm{~A}}^{+}\left(\mathrm{O}^{2-}\right)$ centre.

$4 \mathrm{O}-\mathrm{V}_{\mathrm{A}}$ and $\mathrm{F}_{\mathrm{A}}\left(\mathrm{O}^{2-}\right)$-centres $\mathrm{In}$ the context of the described mechanism it is interesting to calculate some properties of intermediates like $\mathrm{O}-\mathrm{V}_{\mathrm{A}}$ and $\mathrm{F}_{\mathrm{A}}\left(\mathrm{O}^{2-}\right)$-centres. For $\mathrm{O}-\mathrm{V}_{\mathrm{A}}$ configurations $<100>$ and $<110>$ had been calculated $a b$ initio and several more configurations - classically. A $b$ initio barriers for $<100>\rightarrow<110>$ and reverse $<110>\rightarrow<100>$ transformations were 0.49 and $0.30 \mathrm{eV}$, with an energy gain of $0.19 \mathrm{eV}$ for the latter transformation. From classical results it follows that all configurations except the nearest $<100>$ one have almost the same energies, i.e. only $<100>$ configuration is "bonded" $\mathrm{O}-\mathrm{V}_{\mathrm{A}}$, others can be seen as a free vacancy and an isolated $\mathrm{O}^{-}$. That can be understood because the $\mathrm{O}^{\prime}$ ion is not charged with respect to the lattice and there is no long-range coulomb interaction. Therefore it is possible for $\mathrm{O}^{-}-\mathrm{V}_{\mathrm{A}}$ to dissociate at temperatures above $200 \mathrm{~K}$.

The $\mathrm{F}_{\mathrm{A}}\left(\mathrm{O}^{2-}\right)$-centre optical absorption has been calculated. After some correction for the distortion of the strongly delocalised states of the F-centre described elsewhere [10] two absorption bands are obtained: 2.87 and $3.06 \mathrm{eV}$ with oscillator strengths 0.212 and 0.369 , respectively. These results are in agreement with experimental bands at 2.8 and $3.2 \mathrm{eV}$ [9] attributed to the $\mathrm{F}_{\mathrm{A}}\left(\mathrm{O}^{2-}\right)$-centre.

5 Aggregation of oxygen-vacancy dipoles Several configurations of the dimer $\left(\mathrm{O}^{2-}-\mathrm{V}_{\mathrm{A}}\right)_{2}$ were calculated with pair potentials (Fig. 3). The most energetically favourable one is denoted configuration 0 . The association energy of two dipoles is $0.48 \mathrm{eV}$. That has been obtained as the difference between total energies of two remote dipoles and the dimer configuration 0. Energies of other configurations with respect to config. 0 are also shown on Fig. 3. Configurations 1-1, 1-2, 1-3 are formed from configuration 0 when one vacancy switches its position, the rest - when both vacancies do this. The beginning of the dimer decomposition is most likely $0 \rightarrow 1.2 \rightarrow 2.4$ and then one of the oxygen ions in its turn can jump into the vacancy. Calculation of the optical properties of aggregates will provide the data, which can be compared to experiment.

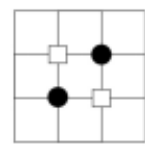

Config. 0 $\mathrm{E}=0.0 \mathrm{eV}$

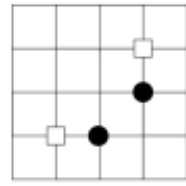

Config. 2-2 $\mathrm{E}=0.595 \mathrm{eV}$

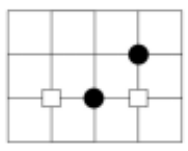

Config. 1-1 $\mathrm{E}=0.375 \mathrm{eV}$

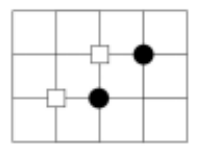

Config. 1-2 $\mathrm{E}=0.361 \mathrm{eV}$

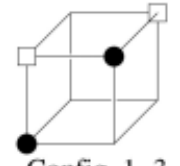

Config. 1-3 $\mathrm{E}=0.307 \mathrm{eV}$

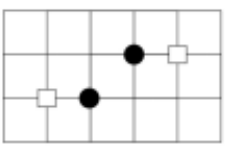

Config. 2-1 $\mathrm{E}=0.538 \mathrm{eV}$
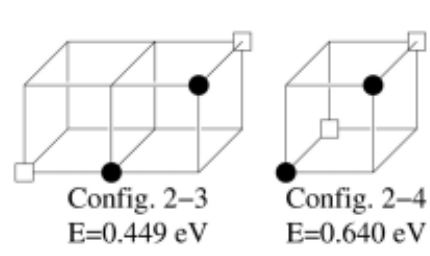

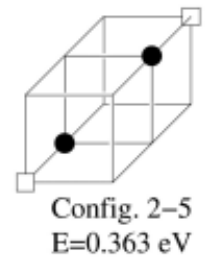

Fig. 3 Configurations of $\left(\mathrm{O}^{2}-\mathrm{V}_{\mathrm{A}}\right)_{2}$ dimer.

\section{References}

[1] V.A. Archangelskaya, V.M. Reyterov, and L.M. Trofimova, Zh. Prikl. Spektroskopii 32, 103 (1980).

[2] E. Radzhabov and P. Figura, phys. stat. sol. (b) 136, K55 (1986).

[3] E. Radzhabov, phys. stat. sol. (b) 123, K79 (1984).

[4] A.V. Puchina, V.E. Puchin, E.A. Cotomin, and M. Reichling, Solid State Commun. 106, 285 (1998). 
[5] P.V. Sushko, A.L. Shluger, and C.R.A. Catlow, Surf. Sci. 450, 153 (2000).

[6] V.B. Sulimov, P.V. Sushko, A.H. Edwards, A.L. Shluger, and A.M. Stoneham, Phys. Rev. B 66, 024108 (2002).

[7] A.S. Mysovsky, P.V. Sushko, S. Mukhopadhyay, A.H. Edwards, and A.L. Shluger, Phys. Rev. B 69, 085202 (2004)

[8] P.W.M. Jacobs and S.H. Ong, J. Phys. Chem. Solids 41, 437 (1979).

[9] R. Rauch and G. Schwotzer, phys. stat. sol. (a) 74, 123 (1982).

[10] A.S. Mysovsky, E.A. Radzhabov, M. Reichling, A.L. Shluger and P.V. Sushko, J. Phys. C, to be published. 